\title{
Asteroid impact, Schumann resonances and the end of dinosaurs
}

\author{
Z. K. Silagadze \\ Budker Institute of Nuclear Physics and Novosibirsk State University, 630 090, \\ Novosibirsk, Russia
}

\begin{abstract}
It is believed that an asteroid/comet impact 65 million years ago ended the dinosaur era. The researchers named the corresponding impact crater Chicxulub, the Mayan word roughly translated as "the dragon's tail." We estimate the expected magnitudes of the Schumann resonance fields immediately after the Chicxulub impact and show that they exceed their present-day values by about $5 \times 10^{4}$ times. Long-term distortion of the Schumann resonance parameters is also expected due to the environmental impact of the Chicxulub event. If Schumann resonances play a regulatory biological role, as some studies indicate, it is possible that the excitation and distortion of Schumann resonances after the asteroid/comet impact was a possible stress factor, which, among other stress factors associated with the impact, contributed to the demise of dinosaurs.

Keywords: Schumann resonances, ELF electromagnetic fields, Chicxulub impact, Dinosaur extinction
\end{abstract}

\section{Introduction}

Dinosaurs have been the dominant group of living organisms on the Earth for over 160 million years (Myr). There were over 1000 species of dinosaurs distributed worldwide. The direct evolutionary descendants of non-avian dinosaurs, birds still make up one of the most proliferate and diverse group of vertebrates. However, non-avian dinosaurs themselves suddenly disappeared about 66 Myr ago [1].

Email address: silagadze@inp.nsk.su ( Z. K. Silagadze) 
There are astounding number and variety of hypotheses about causes of the dinosaur extinction [1, 2]. However, the current research is concentrated around three major ones: 1) an impact of a giant bolid (asteroid or comet) [3, 4, 5]; 2) Volcanic activity in modern-day India's Deccan Traps [6]; 3) Marine regression (drop in sea level) and the corresponding global environmental deterioration [7, 8].

All three of the above stress factors occurred at the end of the Cretaceous, which makes it difficult to disentangle their relative importance in the mass extinction event that occurred at the Cretaceous-Palogene (K-Pg) boundary (formerly Cretaceous-Tertiary or K-T boundary). It is noteworthy that there is little evidence that bolide impacts on the Earth correlate well with episodes of mass extinction other than K-Pg (there have been five mass extinctions in the past $550 \mathrm{Myr}$ ), while both sea regression and massive volcanism do correlate well with such episodes [2].

Nevertheless, modern research [1, 9] found support for a bolide impact as the primary factor of the end-Cretaceous mass extinction. Evidence of the bolid impact, coinciding in time with dinosaur extinction, is ubiquitous, including the huge $150 \mathrm{~km}$ wide Chicxulub crater in the Yucatán Peninsula in Mexico, impact related iridium anomaly worldwide, sediments in various areas of the world dominated by impact melt spherules and an unusually large amount of shocked quartz.

Yet another evidence of the enormous power of the Chicxulub impact has been discovered recently [10]. After the impact, billions of tons of molten and vaporized rock was thrown in all directions. After about ten minutes, these debris reached Tanis, a place at a distance of about $3000 \mathrm{~km}$ from the impact. Bead-sized material, glassy tektites, fell from the sky, piercing everything in its path. Fossil fish at Tanis, densely packed in the deposit, are found with the impact-induced spherules embedded in their gills.

At about the same time, strong seismic waves, generated by the Chicxulub impact, arrived at Tanis generating seiche inundation surge with about $10 \mathrm{~m}$ amplitude [10].

Observations at Tanis expand our knowledge of the destructive effects of the Chicxulub impact, identifying the potential mechanism for sudden and extensive damage to the environment, delivered minutes after the impact to widely separated regions.

Our goal in this note is to show that the global extinction event could have had another very rapidly delivered global precursor, namely the excitation of Schumann resonances with currently unknown but potentially dangerous 
biological effects.

\section{Schumann resonances}

It is useful to introduce the following complex combination of the electric and magnetic fields, called Riemann-Silberstein vector in [11]:

$$
\vec{F}=\sqrt{\frac{2}{\epsilon}}\left(\frac{\vec{D}}{\sqrt{2 \epsilon}}+\frac{\vec{B}}{\sqrt{2 \mu}}\right)=\vec{E}+i c \vec{B},
$$

where $\epsilon$ is the dielectric constant, $\mu$ is the magnetic permeability, and $c=$ $1 / \sqrt{\epsilon \mu}$ is the light velocity in a homogeneous and static medium in which $\vec{D}=\epsilon \vec{E}$ and $\vec{B}=\mu \vec{H}$.

In terms of the Riemann-Silberstein vector, the Maxwell equations read

$$
i \frac{\partial \vec{F}}{\partial t}=c \nabla \times \vec{F}, \quad \nabla \cdot \vec{F}=0 .
$$

It is well known that the process of solving the Maxwell equations can be facilitated by the use of potentials. The Riemann-Silberstein vector can be expressed in terms of the Hertz vector (superpotential) as follows [11]:

$$
\vec{F}=\left[\frac{i}{c} \frac{\partial}{\partial t}+\nabla \times\right](\nabla \times \vec{\Pi}) .
$$

It follows from the first Maxwell equation in (2) that the Hertz superpotential $\vec{\Pi}$ must satisfy the equation

$$
\left(\frac{1}{c^{2}} \frac{\partial^{2}}{\partial t^{2}}-\Delta\right)(\nabla \times \vec{\Pi})=0 .
$$

The name "superpotential" indicates that electric and magnetic fields are expressed through the second derivatives of the superpotential, and not through the first derivatives, as in the case of standard potentials.

\footnotetext{
${ }^{1}$ Maybe we have here an example of the zeroth theorem of the history of science: a discovery (rule, regularity, insight), named after someone, almost always did not originate with that person [12]. There are reasons to believe that this complex vector was first introduced by Heinrich Weber in his 1901 book on the partial differential equations of mathematical physics based on Riemann's lecture notes [13].
} 
Much like more familiar four-potential, Hertz vector is not determined uniquely by (3). In fact the group of gauge transformations of the Hertz vector that leave the Riemann-Silberstein vector unchanged is very large [11, 14]. In particular, when dealing with radiation fields produced by localized sources, a very convenient choice is the assumption that the Hertz superpotential is radial [11]:

$$
\vec{\Pi}(\vec{r}, t)=\vec{r} \Phi(\vec{r}, t) .
$$

Then it follows from (4), since the operator $\hat{\vec{L}}=\vec{r} \times \nabla$ commutes with the Laplacian, that the complex function $\Phi(\vec{r}, t)=U(\vec{r}, t)+i V(\vec{r}, t)$ can be adjusted in such a way using the gauge freedom that it satisfies the wave equation [15, 16]:

$$
\left(\frac{1}{c^{2}} \frac{\partial^{2}}{\partial t^{2}}-\Delta\right) \Phi=0
$$

Using well-known expressions of differential operators in spherical coordinates, we get from (3) and (5)

$$
F_{r}=-\frac{1}{r^{2} \sin \theta} \frac{\partial}{\partial \theta}\left(\sin \theta \frac{\partial(r \Phi)}{\partial \theta}\right)-\frac{1}{r^{2} \sin ^{2} \theta} \frac{\partial^{2}(r \Phi)}{\partial \varphi^{2}},
$$

which by using (6), and assuming harmonic dependence of fields on time of the form $e^{ \pm i \omega t}$, can be transformed into

$$
F_{r}=\left[\frac{\partial^{2}}{\partial r^{2}}+\frac{\omega^{2}}{c^{2}}\right](r \Phi) .
$$

The real and imaginary parts of $\Phi, U$ and $V$ respectively, are called electric and magnetic Debye (super)potentials. They are scalars under the (proper) three-dimensional rotations, but have complicated transformation properties under the Lorentz boosts [15].

Extreme ultraviolet and X-ray radiation from the sun ionizes atoms and molecules in the Earth's upper atmosphere. As a result, at altitudes of several tens of kilometers above the ground, the air conductivity becomes noticeable, and then rapidly increases by six or more orders of magnitude at heights of about $80 \mathrm{~km}$, which indicates the beginning of the ionosphere, a region filled with weakly ionized gas. Thus, a spherical cavity is formed between two relatively good conductors: the lower one is the ground, and the upper one is the ionospheric plasma. 
Depending on the wavelength of the electromagnetic wave, this spherical cavity can be considered as a waveguide, resonator or capacitor [31]. At zero frequency (direct current) we can speak of a spherical capacitor, the upper "electrode" of which carries a potential of $+250 \mathrm{kV}$ relative to the ground. A corresponding electrostatic field, the so-called fair weather field of about 100 $\mathrm{V} / \mathrm{m}$ at the ground, is observed worldwide in local quiet weather conditions.

For radio frequencies with wavelengths much smaller than the Earth's radius, the cavity acts as a waveguide up to frequencies of 10-20 MHz, above which the ionosphere becomes transparent.

Finally, for very low frequency electromagnetic waves with wavelengths comparable to the circumference of the Earth, the Earth-ionosphere cavity can be considered as an electromagnetic resonator, and the corresponding global electromagnetic resonances are called Schumann resonances [31].

More precisely, Schumann resonances are quasi-standing transverse magnetic modes in the Earth-ionosphere cavity, in which the radial component of the magnetic field equals to zero and thus (8) implies $V=0$. Then from (3)

$$
\vec{E}=\nabla \times(\nabla \times \vec{r}) U, \quad \vec{B}=\frac{1}{c^{2}} \frac{\partial}{\partial t} \nabla \times \vec{r} U,
$$

and for the harmonic (complex) fields with $e^{i \omega t}$ time dependence we get

$$
\begin{aligned}
& E_{r}=\left[\frac{\partial^{2}}{\partial r^{2}}+\frac{\omega^{2}}{c^{2}}\right](r U), E_{\theta}=\frac{1}{r} \frac{\partial^{2}(r U)}{\partial r \partial \theta}, E_{\varphi}=\frac{1}{r \sin \theta} \frac{\partial^{2}(r U)}{\partial r \partial \varphi} \\
& B_{\theta}=\frac{i \omega}{c^{2}} \frac{1}{\sin \theta} \frac{\partial U}{\partial \varphi}, B_{\varphi}=-\frac{i \omega}{c^{2}} \frac{\partial U}{\partial \theta}
\end{aligned}
$$

The wave equation (6) for $\mathrm{U}$ (with $e^{i \omega t}$ harmonic time dependence) can be solved by separation of variables in spherical coordinates. Namely, taking $U(\vec{r}, t)=\rho(r) Y_{l m}(\theta, \varphi) e^{i \omega t}$, where $Y_{l m}(\theta, \varphi)$ are spherical functions, and using

$$
\Delta=\frac{1}{r^{2}} \frac{\partial}{\partial r}\left(r^{2} \frac{\partial}{\partial r}\right)+\frac{1}{r^{2}} \Delta_{\perp}
$$

where $\Delta_{\perp}$ is the angular part of the Laplacian with $\Delta_{\perp} Y_{l m}=-l(l+1) Y_{l m}$, we get the spherical Bessel differential equation for the radial function $\rho(r)$ :

$$
\left[\frac{d^{2}}{d r^{2}}+\frac{2}{r} \frac{d}{d r}+k^{2}-\frac{l(l+1)}{r^{2}}\right] \rho(r)=0, \quad k=\frac{\omega}{c} .
$$


Therefore, inside the Earth-ionosphere cavity the Fourier component of $U$ has the form

$$
U(\vec{r}, \omega)=\sum_{l=0}^{\infty} \sum_{m=-l}^{l}\left[A_{l m} h_{l}^{(1)}(k r)+B_{l m} h_{l}^{(2)}(k r)\right] Y_{l m}(\theta, \varphi),
$$

where $h_{l}^{(1)}(k r)$ and $h_{l}^{(2)}(k r)$ are spherical Hankel functions of the first and second kinds, respectively. Since we have chosen $e^{i \omega t}$ for our time evolution, $h_{l}^{(1)}(k r)$ corresponds to the spherical incoming wave, while $h_{l}^{(2)}(k r)$ - to the spherical outgoing wave.

A real Earth-ionosphere waveguide have a very complicated configuration. Here we assume a simplified model [17]. Earth is considered as a perfectly conducting sphere of radius $R$. It is further assumed that the ionosphere begins with an inner radius of $R+h$ and is an infinite, uniform and isotropic plasma with complex dielectric constant (the imaginary part of which is proportional to the plasma conductivity [18]).

In the ionosphere we can have only outgoing spherical waves (Sommerfeld radiation condition [19]). Thus

$$
U(\vec{r}, \omega)=\sum_{l=0}^{\infty} \sum_{m=-l}^{l} C_{l m} h_{l}^{(2)}(k r) Y_{l m}(\theta, \varphi) e^{i \omega t}, \quad r>R+h .
$$

Schumann resonance frequencies are determined by boundary conditions at $r=R$ and $r=R+h$ [17] (for somewhat different approach, see [20]). Namely, at $r=R$ the tangential components of the electric field must vanish. This leads to the condition

$$
\left.\frac{\partial}{\partial r}(r U)\right|_{r=R}=0 .
$$

At $r=R+h$, the tangential components of the electric field $\vec{E}$, and the tangential components of the magnetic field $\vec{H}=\vec{B} / \mu$ must be continuous. If in the cavity $\epsilon \approx \epsilon_{0}, \mu \approx \mu_{0}$, while in the ionosphere $\epsilon=\hat{\epsilon} \epsilon_{0}, \mu \approx \mu_{0}$, in light of (10), the continuity conditions take the form

$$
\begin{gathered}
\left.\frac{\partial}{\partial r}(r U)\right|_{r=(R+h)_{-}}=\left.\frac{\partial}{\partial r}(r U)\right|_{r=(R+h)_{+}}, \\
U\left(r=(R+h)_{-}\right)=\hat{\epsilon} U\left(r=(R+h)_{+}\right) .
\end{gathered}
$$


If we substitute (13) and (14) into (15) and (16), we get a homogeneous system of linear equations

$$
\begin{gathered}
u_{l}^{\prime}(k R) A_{l m}+v_{l}^{\prime}(k R) B_{l m}=0, \\
u_{l}^{\prime}(k(R+h)) A_{l m}+v_{l}^{\prime}(k(R+h)) B_{l m}-v_{l}^{\prime}(\sqrt{\hat{\epsilon}} k(R+h)) C_{l m}=0, \\
u_{l}(k(R+h)) A_{l m}+v_{l}(k(R+h)) B_{l m}-\sqrt{\hat{\epsilon}} v_{l}(\sqrt{\hat{\epsilon}} k(R+h)) C_{l m}=0 .
\end{gathered}
$$

Here we introduced the notations [17]

$$
u_{l}(x)=x h_{l}^{(1)}(x), v_{l}(x)=x h_{l}^{(2)}(x), u_{l}^{\prime}(x)=\frac{d u_{l}(x)}{d x}, v_{l}^{\prime}(x)=\frac{d v_{l}(x)}{d x} .
$$

The system (17) has a non-trivial solution for $A_{l m}, B_{l m}, C_{l m}$, only when the $3 \times 3$ determinant of its coefficients equals zero. This requirement yields the following equation [17]

$$
\begin{aligned}
& u_{l}^{\prime}(k R) v_{l}^{\prime}(k(R+h))-u_{l}^{\prime}(k(R+h)) v_{l}^{\prime}(k R)= \\
& \frac{1}{\sqrt{\hat{\epsilon}}} \frac{v_{l}^{\prime}(\sqrt{\hat{\epsilon}} k(R+h))}{v_{l}(\sqrt{\hat{\epsilon}} k(R+h))}\left[u_{l}^{\prime}(k R) v_{l}(k(R+h))-u_{l}(k(R+h)) v_{l}^{\prime}(k R)\right] .
\end{aligned}
$$

The solutions of this transcendental equation with respect to $k$ determine eigenmodes $\omega=c_{0} k$ of the Earth-ionosphere resonator, which are called Schumann resonances. Here $c_{0}$ is the speed of light inside the resonator, which is the same as the light velocity in vacuum, $c_{0}=1 / \sqrt{\epsilon_{0} \mu_{0}}$, for the approximations used. In general, $\omega$ is a complex number. Its real part gives the eigenfrequency of the resonator, while the imaginary part determines the resonance width, since it corresponds to the damping factor of the eigenmode. The resonance width is characterized by the quality factor $Q=\omega / \Delta \omega$, where $\Delta \omega$ is the resonance width at half maximum.

In the crude approximation of the infinite conductivity of the ionosphere, the right-hand-side of (19) vanishes and the equation for the eigenfrequencies simplifies. Further simplification can be achieved by using $h \ll R$, so that we can expand $u_{l}^{\prime}(k(R+h)) \approx u_{l}^{\prime}(k R)+u_{l}^{\prime \prime}(k R) k h$, and $v_{l}^{\prime}(k(R+h)) \approx$ $v_{l}^{\prime}(k R)+v_{l}^{\prime \prime}(k R) k h$. Besides, it follows from the definitions of $u_{l}(x)$ and $v_{l}(x)$, that

$$
\begin{aligned}
& u_{l}^{\prime}(x)=h_{l}^{(1)}(x)+x \frac{d h_{l}^{(1)}(x)}{d x}, \quad u_{l}^{\prime \prime}(x)=2 \frac{h_{l}^{(1)}(x)}{d x}+x \frac{d^{2} h_{l}^{(1)}(x)}{d x^{2}}, \\
& v_{l}^{\prime}(x)=h_{l}^{(2)}(x)+x \frac{d h_{l}^{(2)}(x)}{d x}, \quad v_{l}^{\prime \prime}(x)=2 \frac{h_{l}^{(2)}(x)}{d x}+x \frac{d^{2} h_{l}^{(2)}(x)}{d x^{2}} .
\end{aligned}
$$


The second derivatives of the Hankel functions can be eliminated by using the fact that $\rho(r)=h_{l}^{(1)}(k r)$ and $\rho(r)=h_{l}^{(2)}(k r)$ satisfy the differential equation (12). This gives

$$
u_{l}^{\prime \prime}(x)=\left(\frac{l(l+1)}{x^{2}}-1\right) x h_{l}^{(1)}(x), \quad v_{l}^{\prime \prime}(x)=\left(\frac{l(l+1)}{x^{2}}-1\right) x h_{l}^{(2)}(x) .
$$

Taking all this into account, in the case of perfectly conducting ionosphere, equation (19) simplifies to

$$
\left(1-\frac{l(l+1)}{k^{2} R^{2}}\right)\left[h_{l}^{(1)}(x) \frac{d h_{l}^{(2)}(x)}{d x}-h_{l}^{(2)}(x) \frac{d h_{l}^{(1)}(x)}{d x}\right]_{x=k R}=0 .
$$

The expression in the square brackets is the Wronskian of $h_{l}^{(1)}(x)$ and $h_{l}^{(2)}(x)$, and it is not zero, because these two solutions of the spherical Bessel equations are independent. Therefore, from (22) we get $\omega_{l}=c_{0} k_{l}=\frac{c_{0}}{R} \sqrt{l(l+1)}$, and in this crude approximation, the Schumann resonance frequencies are

$$
f_{l}=\frac{\omega_{l}}{2 \pi}=\frac{c_{0}}{2 \pi R} \sqrt{l(l+1)}, \quad l=1,2, \ldots
$$

The observed frequencies of the first five Schumann resonances are 7.8, 14.1, 20.3, 26.4 and $32.5 \mathrm{~Hz}$, respectively [21], and they are about $25 \%$ lower than it follows from (23). This discrepancy is due to neglecting the effects of electric losses on the resonance frequencies.

When considering Schumann resonances, there are two sources of losses present [22]. There are losses due to finite electrical conductivity of the atmosphere that increases with altitude, and there are losses due to the fact that both the Earth's surface and the ionosphere are good but imperfect conductors. As is known from a damped harmonic oscillator, the inclusion of the damping effects not only decreases amplitude over time, but also reduces the resonant frequency.

However, more realistic models of ionosphere lead to far more complex theories that in general defy analytical treatment. The ionosphere structures considered include two-layer and multi-layer models, two-exponential, "knee" and "multi-knee" profiles (for relevant references see [27]).

Let us take a quick look at the two-scale-height model of the ionosphere that captures the essential underlying physics of a system with conductivity that increases with height [23, 24, 25]. 
The Joule dissipation responsible for the damping is, in a good approximation, confined in height to two layers. The first, the so-called conduction boundary at the altitude $h_{1}$ is defined by the condition of equality of the displacement and conduction currents, $\sigma_{1}=\epsilon_{0} \omega$, so that at several scale lengths $\left(\xi_{1}\right)$ below $h_{1}$ the atmosphere is insulating, and above several scale lengths it is conducting.

The second, the so-called reflection boundary at $h_{2}$ is the altitude at which $\omega \tau_{D} \approx 1$, where $\tau_{D}$ is the magnetic diffusion time through a conductivity scale length $\xi_{2}$ at $h_{2}$ [24]. In other words, at $h_{2}$ the character of the fields changes from being wavelike to diffusion-like [25]. In terms of conductivity $\sigma_{2}$ at $h_{2}$, this condition has the form $4 \mu_{0} \omega \sigma_{2} \xi_{2}^{2}=1$ [24].

The conductivity profiles in these two characteristic layers of ionosphere are given by exponential functions with different scale lengths $\xi_{1}$ and $\xi_{2}$ : $\sigma(z)=\sigma_{1} \exp \left[\left(z-h_{1}\right) / \xi_{1}\right]$ and $\sigma(z)=\sigma_{2} \exp \left[\left(z-h_{2}\right) / \xi_{2}\right]$ respectively. It can be shown [23, 25] that for such conductivity profiles the real parts of the Schumann resonance eigenfrequencies and the Q-factors are given by

$$
f_{l} \approx \frac{c_{0}}{2 \pi R} \sqrt{l(l+1) \frac{h_{1}}{h_{2}}}, \quad Q_{l} \approx \frac{2}{\pi}\left(\frac{\xi_{1}}{h_{1}}+\frac{\xi_{2}}{h_{2}}\right)^{-1} .
$$

Using typical values of the two-scale-height model $h_{1}=50 \mathrm{~km}, h_{1}=90 \mathrm{~km}$, $\xi_{1}=\xi_{2}=5 \mathrm{~km}$, these expressions yield results that are in quite good agreement with observations [23].

Winfried Otto Schumann, a professor at the Technische Hochschule München, rightfully gets most of the credit for predicting Schumann Resonances. However, Schumann resonance history is an interesting story [26]. The idea of natural global electromagnetic resonances goes back to George F. Fitzgerald in 1893 and Nikola Tesla in 1905 [26, 27]. The formula (23) for resonance frequencies of a spherical condenser was first obtained by Joseph Larmor already in 1894 [26].

Above we have outlined just some basics of Schumann resonances for the reader's convenience. More detailed information about Schumann resonance research can be found in books [17, 28, 29, 30, 31].

\section{Excitation of Schumann resonances by an asteroid impact}

Schumann resonances are excited primarily by lightning discharges. On the other hand, it is known that explosions and hypervelocity impacts are 
accompanied by macroscopic charge separation [32, 33, 34]. Upon a hypervelocity impact, a partially ionized plasma is formed, which rapidly expands. In addition to plasma, the impact will result in the formation of molten and fragmented debris of the target material, which are expected to become negatively charged when in contact with the plasma, since electrons are much more mobile than ions. The subsequent inertial separation of the positively charged plasma and the negatively charged debris will lead to the separation of charge over macroscopic distances [34].

One can also imagine some other mechanisms of charge separation, for example, those that act during the dust storms [35] and volcanic eruptions [36]. Therefore, we assume that an asteroid impact is immediately accompanied by a thunderstorm with a large number of lightning discharges. Namely, let $d N(t)=N e^{-t / T} \frac{d t}{T}$ be the number of lightning discharges during a time period $d t$ at the time $t$ after the impact. Here $T \approx 100 \mathrm{~s}$ is the transient crater formation time for the Chicxulub event [37], and $N$ is the total number of lightning strikes in the impact thunderstorm. If the current in an average individual lightning strike is $I_{0} e^{-t / \tau}$, with $\tau \approx 500 \mu$ s and $I_{0} \approx 2 \cdot 10^{4} \mathrm{~A}$ [38], the total current will be

$$
I(t)=\int_{0}^{t} e^{-\frac{t-s}{\tau}} d N(s)=\frac{N I_{0}}{T} e^{-\frac{t}{\tau}} \int_{0}^{t} e^{\left(\frac{1}{\tau}-\frac{1}{T}\right) s} d s \approx \frac{N I_{0} \tau}{T} e^{-\frac{t}{T}},
$$

where at the last step we have taken into account that $T \gg \tau$ (in fact, this condition is not sufficient do discard the second exponent $e^{-t / \tau}$, which occurs after the integration in (25), since short signals with $\omega_{n} \tau \sim 1$ can excite Schumann resonances just as effectively as long signals with $\omega_{n} T \gg 1$. However, it will be clear from the final answer that we can still neglect the contribution of this term due to the condition $\omega_{n} \tau \ll 1$, which is satisfied by the first few Schumann resonances).

Accordingly, as the current density, which we will consider having only a radial component, we take

$$
j_{r}(\vec{r}, t)=\frac{I(t) \Delta l}{2 \pi r^{2} \sin \theta} \delta(\theta) \delta(r-R) \Theta(t),
$$

where $\Theta(t)$ is the Heaviside step function introduced to indicate that there is no current for $t<0$, and $\Delta l \approx 10^{3} \mathrm{~m} \mathrm{[38]} \mathrm{is} \mathrm{the} \mathrm{length} \mathrm{of} \mathrm{an} \mathrm{average}$ lightning channel. The current density (26), when integrated over the whole space, gives the total current moment: $\int j_{r}(\vec{r}, t) d V=I(t) \Delta l$. 
Now we will consider how the Schumann resonances are excited by the vertical electric dipole with current density (26). We will closely follow [28], for other approaches see [30] and [17, 38].

Maxwell equations

$$
\nabla \times \vec{E}=-\mu_{0} \frac{\partial \vec{H}}{\partial t}, \quad \nabla \times \vec{H}=\epsilon_{0} \frac{\partial \vec{E}}{\partial t}+\vec{j}(\vec{r}, t),
$$

for Fourier components with $e^{i \omega t}$ time dependence take the form

$$
\nabla \times \vec{E}(\vec{r}, \omega)=-i \omega \mu_{0} \vec{H}(\vec{r}, \omega), \quad \nabla \times \vec{H}(\vec{r}, \omega)=i \omega \epsilon_{0} \vec{E}(\vec{r}, \omega)+\vec{j}(\vec{r}, \omega),
$$

where $\vec{j}(\vec{r}, \omega)$ has only the radial component

$$
j_{r}(\vec{r}, \omega)=\int_{-\infty}^{\infty} e^{-i \omega t} j_{r}(\vec{r}, t) d t=\frac{N I_{0} \Delta l \tau}{1+i \omega T} \frac{\delta(\theta) \delta(r-R)}{2 \pi r^{2} \sin \theta} .
$$

A vertical electric dipole source at $\theta=0$ can excite only fields that do not have a $\varphi$-dependence. This follows from the azimuthal symmetry of the problem. Then it can be checked in spherical coordinates that the fields given by equations (10) still satisfy the Maxwell equations (28) if the Debye superpotential $U(r, \theta)$ satisfies the equation

$$
r\left(\Delta+k^{2}\right) U=\left(\frac{\partial^{2}}{\partial r^{2}}+k^{2}\right)(r U)+\frac{1}{r \sin \theta} \frac{\partial}{\partial \theta}\left(\sin \theta \frac{\partial U}{\partial \theta}\right)=-\frac{j_{r}(\vec{r}, \omega)}{i \omega \epsilon_{0}} .
$$

Since $j_{r}(\vec{r}, \omega)$ is proportional to $\delta(r-R)$, it vanishes in the Earth-ionosphere cavity. Thus, in the cavity $U(\vec{r}, \omega)$ is still given by (13) with the difference that only $m=0$ modes are excited due to azimuthal symmetry, and, therefore $Y_{l m}$ spherical functions can be replaced simply by Legendre polynomials $P_{l}(\cos \theta)$ :

$$
U(\vec{r}, \omega)=\sum_{n=0}^{\infty}\left[A_{n} h_{n}^{(1)}(k r)+B_{n} h_{n}^{(2)}(k r)\right] P_{n}(\cos \theta) .
$$

The boundary condition at $r=R+h$ is

$$
\left.\frac{\partial}{\partial r}(r U)\right|_{r=R+h}=0
$$


if the ideally conducting ionosphere is assumed. To get the boundary condition at $r=R$, we integrate (30) over $r$ from $R-\varepsilon$ to $R+\varepsilon$, take into account that inside the ideally conducting Earth there is no tangential electric field and hence $\left.\frac{\partial}{\partial r}(r U)\right|_{r=R-\varepsilon}=0$, and finally take the limit $\varepsilon \rightarrow 0$. As a result, we get [28]

$$
\left.\frac{\partial}{\partial r}(r U)\right|_{r=R}=-\frac{N I_{0} \Delta l \tau}{1+i \omega T} \frac{\delta(\theta)}{2 \pi i \epsilon_{0} \omega R^{2} \sin \theta}=-\sum_{n=0}^{\infty} a_{n} P_{n}(\cos \theta),
$$

where

$$
a_{n}=\frac{N I_{0} \Delta l \tau}{1+i \omega T} \frac{1}{2 \pi i \epsilon_{0} \omega R^{2}}\left(n+\frac{1}{2}\right),
$$

and at the last step, we have expanded $\delta(\theta) / \sin \theta$ into a series of Legendre polynomials:

$$
\frac{\delta(\theta)}{\sin \theta}=\sum_{n=0}^{\infty}\left(n+\frac{1}{2}\right) P_{n}(\cos \theta)
$$

Substituting (31) into (32) and (33), we get the following system of linear equations for unknown coefficients $A_{n}$ and $B_{n}$ :

$$
\begin{aligned}
& A_{n} u_{n}^{\prime}(k(R+h))+B_{n} v_{n}^{\prime}(k(R+h))=0, \\
& A_{n} u_{n}^{\prime}(k R)+B_{n} v_{n}^{\prime}(k R)=-a_{n} .
\end{aligned}
$$

This system is easily solved, and if the results are substituted in (31), we obtain

$$
U=\sum_{n=0}^{\infty} a_{n} \frac{v_{n}^{\prime}(k(R+h)) h_{n}^{(1)}(k r)-u_{n}^{\prime}(k(R+h)) h_{n}^{(2)}(k r)}{u_{n}^{\prime}(k(R+h)) v_{n}^{\prime}(k R)-v_{n}^{\prime}(k(R+h)) u_{n}^{\prime}(k R)} P_{n}(\cos \theta) .
$$

From (12) It follows that the Hankel functions satisfy the relation

$$
\left(\frac{d^{2}}{d r^{2}}+k^{2}\right)\left(r h_{n}^{(1,2)}(k r)\right)=\frac{n(n+1)}{r} h_{n}^{(1,2)}(k r) .
$$

Then from (10) and (37) we obtain the following expression for the Fourier component $E_{r}(\vec{r}, \omega)$ of the electric field on the ground (at $r=R$ ):

$$
E_{r}(\vec{r}, \omega)=\sum_{n=0}^{\infty} \frac{a_{n} n(n+1)}{R} c_{n} P_{n}(\cos \theta)
$$


where

$$
c_{n}=\frac{v_{n}^{\prime}(k(R+h)) h_{n}^{(1)}(k R)-u_{n}^{\prime}(k(R+h)) h_{n}^{(2)}(k R)}{u_{n}^{\prime}(k(R+h)) v_{n}^{\prime}(k R)-v_{n}^{\prime}(k(R+h)) u_{n}^{\prime}(k R)} .
$$

Now we use, as in the previous section, smallness of the ratio $h / R$ and expand both the numerator and denominator of $c_{n}$ in terms of this small quantity. To first order, we have

$$
\begin{aligned}
& u_{n}^{\prime}(k(R+h)) v_{n}^{\prime}(k R)-v_{n}^{\prime}(k(R+h)) p u_{n}^{\prime}(k R) \approx k h\left(n(n+1)-k^{2} R^{2}\right) W, \\
& v_{n}^{\prime}(k(R+h)) h_{n}^{(1)}(k R)-u_{n}^{\prime}(k(R+h)) h_{n}^{(2)}(k R) \approx k R W,
\end{aligned}
$$

where $W$ is the Wronskian of $h_{l}^{(1)}(x)$ and $h_{l}^{(2)}(x)$ at $x=k R$. Replacing $n(n+1)$ by $\frac{R^{2}}{c^{2}} \omega_{n}^{2}$, and $k^{2}$ by $\frac{\omega^{2}}{c^{2}}$, we get

$$
E_{r}(\vec{r}, \omega)=\sum_{n=0}^{\infty} \frac{a_{n} \omega_{n}^{2} P_{n}(\cos \theta)}{h\left(\omega_{n}^{2}-\omega^{2}\right)}=\frac{N I_{0} \Delta l \tau}{2 \pi i \epsilon_{0} \omega R^{2} h(1+i \omega T)} \sum_{n=0}^{\infty} \frac{\omega_{n}^{2}\left(n+\frac{1}{2}\right) P_{n}(\cos \theta)}{\omega_{n}^{2}-\omega^{2}} .
$$

But $\frac{\omega_{n}^{2}}{\omega_{n}^{2}-\omega^{2}}=1+\frac{\omega^{2}}{\omega_{n}^{2}-\omega^{2}}$, and the first term according to (35) will lead to a $\delta(\theta)$ proportional contribution that is equal to zero outside the source. Therefore, finally we can write

$$
E_{r}(\vec{r}, \omega)=\frac{N I_{0} \Delta l \tau}{4 \pi i \epsilon_{0} R^{2} h(1+i \omega T)} \sum_{n=0}^{\infty} \frac{\omega}{\omega_{n}^{2}-\omega^{2}}(2 n+1) P_{n}(\cos \theta) .
$$

To find the electric field in the time domain, we perform the inverse Fourier transform of the frequency domain field $E_{r}(\vec{r}, \omega)$ (since it is assumed that the Earth is perfectly conductive, the electric field on the ground is radial, so we omit the lower index indicating the radial component in $E(\vec{r}, t))$ :

$$
E(\vec{r}, t)=\frac{1}{2 \pi} \int_{-\infty}^{\infty} e^{i \omega t} E_{r}(\vec{r}, \omega) d \omega
$$

However, for the integral (44) to have a well-defined meaning, it is necessary to indicate how to handle the singularities of the integrand: as is clear from (43), we have three simple poles at $\pm \omega_{n}$ and $\frac{i}{T}$, and the first two of them lie on the integration contour of (44).

This problem is solved by noting that in reality the Earth and ionosphere are not ideal conductors and as a result the Schumann eigenfrequencies become complex with small imaginary parts $\gamma_{n}=\frac{\omega_{n}}{2 Q_{n}} \ll \omega_{n}$ [17] (the quality factors for the first Schumann resonances are $Q_{1} \approx 4.63, Q_{2} \approx 5.96$, 
$\left.Q_{3} \approx 6.56, Q_{4} \approx 6.83, Q_{5} \approx 6.95[17]\right)$. For a dissipative ionosphere, the imaginary part $\gamma_{n}$ of the positive pole at $\omega=\omega_{n}$ is positive. The imaginary part of the negative pole at $\omega=-\omega_{n}$ is fixed by the condition $E_{r}^{*}(\vec{r}, \omega)=E_{r}(\vec{r},-\omega)$ (the reality condition for the time domain field $\left.E_{r}(\vec{r}, t)\right)$ and turns out to be also $\gamma_{n}$. Therefore, we replace $\left(\omega^{2}-\omega_{n}^{2}\right)^{-1}$ in the integral (44) by $\left[\left(\omega-\omega_{n}-i \gamma_{n}\right)\left(\omega+\omega_{n}-i \gamma_{n}\right)\right]^{-1}$, close the integration contour in the upper half-plane where the integrand decreases exponentially, and evaluate the integral according to the Cauchy residue theorem as a sum of residues at three simple poles. As a result, we obtain

$$
E(\vec{r}, t)=\frac{N I_{0} \Delta l \tau}{4 \pi \epsilon_{0} R^{2} h} \sum_{n=0}^{\infty} \frac{(2 n+1) P_{n}(\cos \theta)}{1+\omega_{n}^{2} T^{2}}\left[e^{-\frac{t}{T}}-e^{-\gamma_{n} t}\left(\cos \omega_{n} t+\omega_{n} T \sin \omega_{n} t\right)\right] .
$$

The first $e^{-t / T}$ term in square braces expresses the direct, non-resonant contribution to the electric field from the source, while the remaining terms correspond to the excitation of resonant modes of the cavity [38]. Since $\omega_{n} T \gg 1$, the resonant part of the electric field takes the form

$$
E_{r e s}(\vec{r}, t)=-\frac{N I_{0} \Delta l \tau}{4 \pi \epsilon_{0} R^{2} h} \sum_{n=0}(2 n+1) \frac{e^{-\gamma_{n} t}}{\omega_{n} T} \sin \omega_{n} t P_{n}(\cos \theta) .
$$

To estimate an average amplitude of the excitation, we replace $e^{-\gamma_{n} t}$ by its average value $\frac{1}{T} \int_{0}^{T} e^{-\gamma_{n} t} d t \approx \frac{1}{\gamma_{n} T}[38]$. In this way, we get for the amplitude of the first Schumann resonance

$$
\mathcal{A}_{1} \approx \frac{3 N I_{0} \Delta l \tau}{4 \pi \epsilon_{0} R^{2} h \omega_{1} \gamma_{1} T^{2}}=\frac{3 \Delta Q \Delta l}{4 \pi \epsilon_{0} R^{2} h \omega_{1} \gamma_{1} T^{2}},
$$

where $\Delta Q=N I_{0} \tau$ is the total amount of electric charge separated by a macroscopic distance. In [34] the following empirical relation was obtained for $\Delta Q$ in laboratory scale hypervelocity impacts (all quantities are in the SI units)

$$
\Delta Q \approx 10^{-2} m\left(\frac{V}{3000}\right)^{2.6 \pm 0.1}
$$

where $m$ is the impactor mass, and $V$ is its velocity. It was argued [39] that the Chicxulub impactor was a fast asteroid or a long-period comet with energy between $1.3 \times 10^{24} \mathrm{~J}$ and $5.8 \times 10^{25} \mathrm{~J}$, and mass between $1.0 \times 10^{15} \mathrm{~kg}$ and $4.6 \times 10^{17} \mathrm{~kg}$. Taking the lowest numbers $m=1.0 \times 10^{15} \mathrm{~kg}$ and $E_{k i n}=$ 
$1.3 \times 10^{24} \mathrm{~J}$, for the velocity we obtain $V=\sqrt{\frac{2 E_{k i n}}{m}} \approx 50 \mathrm{~km} / \mathrm{s}$. Then an interpolation of empirical relation (48) to this enormous scale gives a huge number $\Delta Q \approx 1.5 \times 10^{16} \mathrm{C}$. However, a recent simulation [40] resulted in the Chicxulub scale impact-generated magnetic field that was three orders of magnitude smaller than expected from the relation (48). Therefore, as a more realistic estimate, we will take $\Delta Q \approx 1.5 \times 10^{13} \mathrm{C}$. As for other parameters in (47), we will assume $R=6400 \mathrm{~km}, h=75 \mathrm{~km}, \omega_{1}=49$ and $\gamma_{1}=5.3$. Then we get from (47) the following amplitudes for the electric and magnetic fields of the first Schumann resonance:

$$
\mathcal{A}_{1} \approx 50 \mathrm{~V} / \mathrm{m}, \quad \mathcal{B}_{1}=\frac{\mathcal{A}_{1}}{V_{p h}} \approx 230 \mathrm{nT},
$$

where $V_{p h} \approx 0.7 c_{0}$ is the phase velocity of the electromagnetic waves in the earth-ionosphere cavity. For comparison, the measured Schumann resonance background fields are very small, of the order of $\mathrm{mV} / \mathrm{m}$ for the electric field, and several pT for the magnetic field [27]. As we see, estimated magnitudes of the Chicxulub impact induced Schumann resonance fields exceed to their present-day values about $5 \times 10^{4}$ times.

\section{On biological effects of ELF electromagnetic fields}

Shortly after Schumann and his graduate student König made their first attempts to detect Schumann resonances, König and Ankermüller noted a striking similarity between these signals and human brain electroencephalograms (EEG) [41].

The classical EEG rhythms are delta (1-3 Hz), theta (4-7 Hz), alpha (8-13 $\mathrm{Hz})$, beta $(14-29 \mathrm{~Hz})$ and gamma $(30-80+\mathrm{Hz})$ [42], and we can try to roughly estimate these fundamental brain frequencies as follows [43].

Human neocortex, which form most of the white matter, contains about $10^{10}$ interconnected neurons. Imagine that the wrinkled surface of each hemisphere, where these neurons are situated, is inflated so that to create a spherical shell with effective radius $a=\sqrt{S / 4 \pi}$, where $S=1000-1500 \mathrm{~cm}^{2}$ is the surface area of the hemisphere. Characteristic corticocortical axon excitation propagation speed is $V=600-900 \mathrm{~cm} / \mathrm{s}$. Therefore we can write the wave equation for the propagation of these excitation waves on the surface of the sphere as follows:

$$
\Delta \Phi(\theta, \varphi, t)=\frac{1}{V^{2}} \frac{\partial^{2} \Phi(\theta, \varphi, t)}{\partial t^{2}}
$$


where $\Phi$ is some quantity characterizing the excitation. Because of spherical symmetry, we seek the solution of (50) in the form

$$
\Phi(\theta, \varphi, t)=\sum_{l=0}^{\infty} \sum_{m=-l}^{l} A_{l m} F_{l}(t) Y_{l m}(\theta, \varphi) .
$$

Recalling (11) and taking into account that $r=a=$ const and $\Delta_{\perp} Y_{l m}=$ $-l(l+1) Y_{l m}$, we get the following differential equation for $F_{l}(t)$ after separating the variables:

$$
\frac{1}{V^{2}} \frac{d^{2} F_{l}(t)}{d t^{2}}=-\frac{l(l+1)}{a^{2}} F_{l}(t) .
$$

This is the equation of harmonic oscillations with the cyclic frequency

$$
\omega_{l}=\frac{V}{a} \sqrt{l(l+1)} .
$$

In particular, for the first fundamental frequency we get $f_{1}=\frac{\omega_{1}}{2 \pi}=8-18 \mathrm{~Hz}$, which is close to the frequency of alpha rhythm [43].

From how we obtained Schumann resonances and brain waves, it should be clear that the similarities between them are the result of spherical symmetry and the small height of the ionosphere compared to the radius of the Earth. The existence of standing waves requires only that the material medium supports traveling waves that do not decay too quickly. Then the corresponding resonant frequencies are determined from the geometry of the problem and from the boundary conditions. Therefore any similarity between brain waves and Schumann resonances may well be just a coincidence, and for their interconnection a wild stretch of our imagination will be required [44]. Nevertheless, some arguments can be envisaged that these two desperately different phenomena are actually interrelated.

ELF electromagnetic fields and Schumann resonances have been present on Earth since the formation of the ionosphere. Therefore, they accompanied life from the very beginning, and it does not seem too wild to assume that in the course of evolution living organisms have found some useful application to these ubiquitous electromagnetic fields. One can even imagine that the ELF electromagnetic fields and related electric activity in the Precambrian Earth's atmosphere played the crucial role in the emergence of life according to the following scenario [45, 46]. 
In the Precambrian era, the atmosphere of the Earth was much larger and more similar to what Jupiter has today. In addition, the ionosphere was also much farther than today, about $10^{3} \mathrm{~km}$ far from the Earth's surface, in the immediate vicinity of the Van Allen belts. As a result, fluctuations of current in the Van Allen belts were capably of generating huge currents in the nearby ionosphere and the coupling of these currents to the Earth's metallic core would lead to an enormous and constant electrical activity. It is believed [45], these electrical discharges were essential for production of amino acids and peptides from which the first living organisms were formed. This process was accompanied by an intense background of the ELF electromagnetic field, which could affect the formation and functionality of the first living cells and organisms.

It has been suggested that these atmospheric ELF background fields played a major role in the evolution of biological systems, especially in the early stages of evolution [47]. In particular, the dominant brain wave frequencies may be the evolutionary result of the presence and effect of this ELF electromagnetic background [48]. This idea is to some extent supported by the amazing fact that many species exhibit, irrespective of the size and complexity of their brain, essentially similar low-frequency electrical activity [47].

Various remote sensing systems of living organisms, such as visual system or the infrared sensors of snakes, have been developed due to the presence of electromagnetic energy in the corresponding parts of the spectrum. On early Earth, there was a significant amount of electromagnetic energy in the ELF portion of the spectrum. Thus, we can expect that organisms could adapt and somehow use this part of the electromagnetic spectrum too, in particular the Schumann peaks of the Earth's ELF electromagnetic field [48]. The following observation provides some support for this idea.

Heat shock genes are responsible for adapting organisms to harsh environmental conditions. They are ubiquitous, present in various organisms from bacteria to humans and represent the most conservative and ancient group of genes. The proteins encoded by these genes (heat shock proteins, HSPs) serve as molecular chaperones, which help in the repair, folding and assembly of nascent proteins during stress and prevent the accumulation of damaged cellular proteins.

It has been experimentally demonstrated that the ELF electromagnetic fields can induce various heat shock proteins and, in particular, HSP70, like a real heat shock. The most surprising fact was that the electromagnetic 
fields caused the synthesis of HSP70 at an energy density of fourteen orders of magnitude lower than in heat shock [49].

Such extraordinary sensitivity to the ELF magnetic fields (unlike ELF electric fields, magnetic fields easily penetrate biological tissues) should have a good evolutionary basis. Astrophysical simulations show that shortly after the formation of the solar system, giant planets Jupiter and Saturn begin to migrate inward or outward. This planetary migration destabilizes the orbits of Neptune and Uranus into eccentric ellipses. As a result of this, the ice giants begin to cross the planetesimal disk beyond the orbit of Neptune and gravitationally scatter these planetesimals, forcing many of them to go along the Earth-crossing trajectories. The resulting so-called Late Heavy Bombardment (LHB) could have both positive and negative consequences for the emergence of life [50]. In any case, the first living cells are expected to face grave dangers of powerful bombardment by meteorites (LHB tail). Thus, it can be assumed that the cells could use ELF magnetic pulses as a kind of early warning system that gives them time to prepare for other really dangerous stressors such as the heat pulse and the blast wave, which often follow the electromagnetic pulse [51].

However, a very detailed analysis in [52] indicates that, from the point of view of the conventional classical physics, it remains a mystery that very weak ELF electromagnetic fields can cause any biological effect at all. The problem is the thermal noise. If we assume that random electric fields in biological tissues generated by thermal fluctuations of charge densities are correctly described by the Johnson-Nyquist formula, as in ordinary conductors, then the inevitable conclusion is that, for external ELF electric fields weaker than $300 \mathrm{~V} / \mathrm{m}$, and for external ELF magnetic fields weaker than $50 \mu \mathrm{T}$, it seems impossible to influence biological processes, since any effects generated by such fields in the body will be masked by thermal noise [52]. This objection is known as the $\mathrm{kT}$ problem.

Despite of these categorical conclusions, biologists continued the experimental attempts to detect biological effects of ELF electromagnetic fields, remembering the words of Szent-Gyorgyi that "the biologist depends on the judgment of the physicist, but must be rather cautious when told that this or that is improbable" [53].

As a result of these attempts, a diverse and incontrovertible evidence had been accumulated indicating that ELF radiation has important effects on the functioning of cells [54]. We cite only a few reviews of the subject $[55,56,57,58,59,60]$, where further references can be found. 
The usual formulation of the $\mathrm{kT}$ problem is based on several implicit assumptions that are not always justified [61, 62]. For example, the JohnsonNyquist formula assumes that the system is in a thermal equilibrium. Even so, although a living organism as a whole is very far from thermal equilibrium, the nature of this non-equilibrium is such that the concept of a well-defined temperature nevertheless exists [63]. In fact, the physical nature of the biological effects of ELF fields still remains an enigma, and more work is needed to elucidate the comprehensive mechanisms behind these effects [60, 63].

\section{Concluding remarks}

As we have seen, the magnitudes of the Schumann resonance fields are expected to increase tremendously after the Chicxulub impact. Nevertheless, this effect will be rather short-lived, since Schumann resonance fields decay rapidly due to low Q-factors of resonances.

In the long run, the impact of this magnitude will cause a very serious environmental damage. As a result, stratospheric dust, sulfates released as a result of impact, and soot from extensive worldwide forest fires caused by exposure to the impact related thermal radiation, can lead to significant climate changes over decades (impact winter) [64] and hence modify lightning activity, which is the main source of energy for Schumann resonances.

In addition, blast wave for some time distorts the ionosphere and changes the frequencies of Schumann resonances. It is clear from (24) that not only the frequency of the first Schumann resonance will be changed, but all other Schumann resonances too. However, it is difficult to estimate the magnitude of the effect without detailed modeling of the expected impact on the ionosphere. Note that after high altitude "Starfish" nuclear test explosion, all resonance frequencies abruptly dropped by about $0.5 \mathrm{~Hz}$ [31].

It has been suggested that ELF background atmospheric fields played a major role in the evolution of biological systems, and in particular that Schumann resonances are used for synchronization by living organisms [47, 65]. If so, then the abrupt change in the Schumann resonance parameters after the Chicxulub impact could have a stressful effect, contributing to a devastating load on the global biosphere, including dinosaurs.

A somewhat similar idea can be found in [66], where it was suggested that the influence of the ELF and ultra-law frequency (ULF) electromagnetic fields produced by widespread earthquakes and volcanism in the dinosaur era stimulated their growth in size, and when these phenomena were no longer 
so common dinosaurs became extinct for a number of reasons, including the loss of intensity of the ULF/ELF electromagnetic fields.

In addition, we note that the possible abrupt distortion of the Schumann resonance parameters after a full-scale nuclear war is an additional risk factor that has not been studied, and, therefore, the danger that humanity will become extinct like dinosaurs after such a catastrophic event is perhaps underestimated by politicians.

\section{Acknowledgments}

The work is supported by the Ministry of Education and Science of the Russian Federation. The author is grateful to the anonymous reviewer for constructive suggestions, which helped to improve this paper.

\section{References}

[1] S. L. Brusatte et al., The extinction of the dinosaurs, Biol. Rev. 90 (2015), 628-642. https://doi.org/10.1111/brv.12128

[2] J. D. Archibald, Dinosaur Extinction: Past and Present Perceptions, in M. K. Brett-Surman, T. R. Holtz, J. O. Farlow and B. Walter (eds.) The Complete Dinosaur, Second Edition (Indiana Press: Bloomington, 2012), pp. 1027-1038.

[3] M. W. De Laubenfels, Dinosaur Extinctions: One More Hypothesis, J. Paleontology 30 (1956), 207-218.

[4] L. W. Alvarez, W. Alvarez, F. Asaro and H. V. Michel, Extraterrestrial cause for the Cretaceous-Tertiary extinction, Science 208(1980), 10951108. https://doi.org/10.1126/science.208.4448.1095

[5] W. Alvarez, T. rex and the Crater of Doom (Princeton University Press: Princeton, 1997).

[6] D. M. McLean, A terminal Mesozoic "Greenhouse": lessons from the past, Science 201 (1978), 401-406. https://doi.org/10.1126/science.201.4354.401

[7] M. R. Cooper, Eustacy during the Cretaceous: Its implications and importance, Palaeogeogr., Palaeoclimatol., Palaeoecol. 22 (1977), 1-60. https://doi .org/10.1016/0031-0182(77)90032-3 
[8] D. A. Russell, The Enigma of the Extinction of the Dinosaurs, Ann. Rev. Earth Planet. Sci. 7 (1979), 163-182. https://doi.org/10.1146/annurev.ea.07.050179.001115

[9] P. M. Hull et al., On impact and volcanism across the Cretaceous-Paleogene boundary, Science 367 (2020), 266-272. https://doi.org/10.1126/science.aay5055

[10] R. A. DePalma et al., A seismically induced onshore surge deposit at the KPg boundary, North Dakota, PNAS 116 (2019), 8190-8199. https://doi.org/10.1073/pnas.1817407116

[11] I. Bialynicki-Birula and Z. Bialynicka-Birula, The role of the Riemann-Silberstein vector in classical and quantum theories of electromagnetism, J. Phys. A: Math. Theor. 46 (2013), 053001. https://doi .org/10.1088/1751-8113/46/5/053001 Corrigendum: J. Phys. A: Math. Theor. 46 (2013), 159501.

[12] J. D. Jackson, Examples of the Zeroth Theorem of the History of Physics, Am. J. Phys. 76 (2008), 704-719. https://doi.org/10.1119/1.2904468

[13] M. K. H. Kiessling and A. S. Tahvildar-Zadeh, On the quantummechanics of a single photon, J. Math. Phys. 59 (2018), 112302. https://doi.org/10.1063/1.5021066

[14] A. Nisbet, Hertzian electromagnetic potentials and associated gauge transformations, Proc. R. Soc. Lond. A 231 (1955), 250-263. https://doi.org/10.1098/rspa.1955.0170

[15] D. K. Monroe, Lorentz transformation of Debye potentials, J. Math. Phys. 5 (1984), 1787-1790. https://doi.org/10.1063/1.526355

[16] D. S. Jones, The theory of electromagnetism (Pergamon Press: Oxford, 1964).

[17] P. Bliokh, A. Nickolaenko and Yu. Filippov, Schumann resonances in the earth-ionosphere cavity (Peter Peregrinus: London, 1980).

[18] M. Born and E. Wolf, Principles of Optics (Pergamon: Oxford, 1980). 
[19] S. H. Schot, Eighty years of Sommerfeld's radiation condition, Hist. Math. 19 (1992), 385-401. https://doi.org/10.1016/0315-0860(92)90004-U

[20] M. F. Ciappina and M. Febbo, Schumann's resonances: A particular example of a spherical resonant cavity, Am. J. Phys. bf 72 (2004), 704709. https://doi.org/10.1119/1.1652037

[21] M. Balser and C. A. Wagner, Observations of EarthIonosphere Cavity Resonances, Nature 188 (1960), 638-641. https://doi.org/10.1038/188638a0

[22] J. A. Morente et al., Do Schumann resonance frequencies depend on altitude?, J. Geophys. Res. 109 (2004), A05306. https://doi.org/10.1029/2003JA010305

[23] D. D. Sentman, Schumann resonance spectra in a two-scale-height Earth-ionosphere cavity, J. Geophys. Res. 101 (1996), 9479-9487. https://doi.org/10.1029/95JD03301

[24] C. Greifinger and P. Greifinger, Approximate method for determining ELF eigenvalues in the earth-ionosphere cavity, Radio Sci. 13 (1978), 831-837. https://doi.org/10.1029/RS013i005p00831

[25] D. D. Sentman, Approximate Schumann resonance parameters for a two-scale-height ionosphere, J. Atmos. Terr. Phys. 52 (1990), 35-46. https://doi.org/10.1016/0021-9169(90)90113-2

[26] B. P. Besser, Synopsis of the historical development of Schumann resonances, Radio Science 42 (2007), RS2S02. https://doi.org/10.1029/2006RS003495

[27] C. Price, ELF Electromagnetic Waves from Lightning: The Schumann Resonances, Atmosphere 7 (2016), 116. https://doi.org/10.3390/atmos7090116

[28] V. Surkov and M. Hayakawa, Ultra and Extremely Low Frequency Electromagnetic Fields (Springer: Tokyo, 2014).

[29] K. Rawer, Wave Propagation in the Ionosphere (Springer Science + Business Media: Dordrecht, 1993). 
[30] J. R. Wait, Electromagnetic Waves in Stratified Media (Pergamon Press: Oxford, 1970).

[31] A. Nickolaenko and M. Hayakawa, Schumann Resonance for Tyros (Springer: Tokyo, 2014).

[32] S. P. Soloviev and J. J. Sweeney, Generation of electric and magnetic field during detonation of high explosive charges in boreholes, J. Geophys. Res. 110 (2005), B01312. https://doi.org/10.1029/2004JB003223

[33] V. V. Adushkin and S. P. Soloviev, Generation of low-frequency electric fields by explosion crater formation, J. Geophys. Res. 101 (1996), 20,165-20,173. https://doi.org/10.1029/96JB00952

[34] D. A. Crawford and P. H. Schultz, Electromagnetic properties of impactgenerated plasma, vapor and debris, Intern. J. Impact Eng. 23 (1999), 169-180. https://doi.org/10.1016/S0734-743X (99)00070-6

[35] T. Pähtz, H. J. Herrmann and T. Shinbrot, Why do particle clouds generate electric charges?, Nature Phys. 6 (2010), 364-368. https://doi.org/10.1038/nphys1631

[36] P. Arason, A. J. Bennett and L. E. Burgin, Charge mechanism of volcanic lightning revealed during the 2010 eruption of Eyjafjallajökull, J. Geophys. Res. bf 116 (2011), B00C03. https://doi.org/10.1029/2011JB008651

[37] E. P. Turtle et al., Impact structures: What does crater diameter mean?, in T. Kenkmann, F. Hörz, and A. Deutsch (eds.), Large meteorite impacts III: Geological Society of America Special Paper 384, pp. 1-24, 2005.

[38] D. D. Sentman, Excitation of the Earth-Ionosphere Cavity by an Underground Nuclear Test, Appendix B in J. J. Sweeney, An Investigation of the Usefulness of Extremely Low-Frequency Electromagnetic Measurements for Treaty Verification, Lawrence Livermore Laboratory report UCRL-53899, Livermore, 1989.

[39] H. J. Durand-Manterola and G. Cordero-Tercero, Assessments of the energy, mass and size of the Chicxulub Impactor, arXiv:1403.6391. 
[40] D. A. Crawford, Simulations of magnetic fields produced by asteroid impact: Possible implications for planetary paleomagnetism, Intern. J. Impact Eng. 137 (2020), 103464. https://doi.org/10.1016/j.ijimpeng.2019.103464

[41] H. König and F. Ankermüller, Über den Einfluß besonders niederfrequenter elektrischer Vorgänge in der Atmosphäre auf den Menschen, Naturwissenschaften 47 (1960), 486-490. https://doi.org/10.1007/BF00631044

[42] T. H. Bullock, Biology of brain waves: natural history and evolution of an information-rich sign of activity, in K. Arikan, N. Moore (Eds.), Advances in Electrophysiology in Clinical Practice and Research (Kjellberg: Wheaton, 2002).

[43] P. L. Nunez, Towards a physics of neocortex, in V. Z. Marmarelis (Ed.), Advanced Methods of Physiological System Modeling (Plenum Press: New York, 1989).

[44] P. L. Nunez, Brain, Mind, and the Structure of Reality (Oxford University Press: Oxford, 2010).

[45] F. E. Cole and E. R. Graf, Precambrian ELF and Abiogenesis, in M. A. Persinger (Ed.), ELF and VLF Electromagnetic Field Effects (Springer: Boston, 1974), pp. 243-274. https://doi.org/10.1007/978-1-4684-9004-6_9

[46] R. O. Becker and G. Selden, The Body Electric: Electromagnetism and the Foundation of Life (Morrow: New York, 1985).

[47] C. Price, E. Williams, G. Elhalel and D. Sentman, Natural ELF fields in the atmosphere and in living organisms, Int. J. Biometeorol. 65 (2021), 85-92 (2021). https://doi.org/10.1007/s00484-020-01864-6

[48] L. K. Direnfeld, The Genesis of the EEG and its Relation to Electromagnetic Radiation, J. Bioelectricity 2 (1983), 111-121. https://doi.org/10.3109/15368378309009845

[49] R. Goodman and M. Blank, Insights Into Electromagnetic Interaction Mechanisms, J. Cell Physiol. bf 192 (2002), 16-22. https://doi.org/10.1002/jcp.10098 
[50] B. K. D. Pearce, A. S. Tupper, R. E. Pudritz and P. G. Higgs, Constraining the Time Interval for the Origin of Life on Earth, Astrobiology bf 18 (2018), 343-364. https://doi.org/10.1089/ast.2017.1674

[51] Z. K. Silagadze, Tunguska genetic anomaly and electrophonic meteors, Acta Phys. Polon. B bf 36 (2005), 935-964.

[52] R. K. Adair, Constraints on biological effects of weak extremely-lowfrequency electromagnetic fields, Phys. Rev. A 43 (1991), 1039-1048. https://doi.org/10.1103/physreva.43.1039

[53] A. S. Presman, it Electromagnetic Fields and Life (Springer Science+Business Media: New York, 1970).

[54] W. R. Adey, The energy around us, The Sciences 26 (N1) (1986), 52-58. https://doi.org/10.1002/j.2326-1951.1986.tb02827.x

[55] R. H. W. Funk, T. Monsees and N. Özkucur, Electromagnetic effects - From cell biology to medicine, Progress in Histochemistry and Cytochemistry 43 (2009), 177-264. https://doi.org/10.1016/j.proghi.2008.07.001

[56] V. N. Binhi and A. V. Savin, Effects of weak magnetic fields on biological systems: physical aspects, Phys. Usp. 46 (2003), 259-291. https://doi.org/10.1070/PU2003v046n03ABEH001283

[57] G. J. Beers, Biological Effects of Weak Electromagnetic Fields From 0 Hz to 200 MHz: A Survey of the Literature With Special Emphasis on Possible Magnetic Resonance Effects, Magn. Reson. Imaging 7 (1989), 309-31. https://doi .org/10.1016/0730-725x (89)90556-0

[58] M. A. Persinger, H. W. Ludwig and K-P. Ossenkopp, Psychophysiological Effects of Extremely Low Frequency Electromagnetic Fields: A Review, Percept. Motor Skills bf 36 (1973), 1131-1159. https://doi.org/10.2466/pms.1973.36.3c.1131

[59] N. G. Ptitsyna, G. Villoresi, L. I. Dorman, N. Iucci and M. I. Tyasto, Natural and man-made low-frequency magnetic fields as a potential health hazard, Phys. Usp. bf 41 (1998), 687-709. https://doi.org/10.1070/PU1998v041n07ABEH000419 
[60] A. Karimi, F. Ghadiri Moghaddam and M. Valipour, Insights in the biology of extremely low-frequency magnetic fields exposure on human health, Mol. Biol. Rep. 47 (2020), 5621-5633. https://doi.org/10.1007/s11033-020-05563-8

[61] V. N. Binhi and A. B. Rubin, Magnetobiology: The kT Paradox and Possible Solutions, Electromagnetic Biol. Med. 26 (2007), 45-62. https://doi.org/10.1080/15368370701205677

[62] M. Scalia, M. Sperini and F. Guidi, The Johnson Noise in Biological Matter, Math. Prob. Eng. 2012, 582126. https://doi.org/10.1155/2012/582126

[63] A. Yu. Grosberg, A few remarks evoked by Binhi and Savin's review on magnetobiology, Phys. Usp. 46 (2003), 1113-1116. https://doi.org/10.1070/PU2003v046n10ABEH001633; V. N. Binhi and A. V. Savin, Reply to A. Yu. Grosberg's letter to the Physics-Uspekhi Editorial Board, Phys. Usp. 48 (2005), 537-538. https://doi.org/10.1070/PU2005v048n05ABEH002049

[64] O. B. Toon, K. Zahnle, D. Morrison, R. P. Turco and C. Covey, Environmental perturbations caused by the impacts of asteroids and comets, Rev. Geophys. 35 (1997), 41-78. https://doi.org/10.1029/96RG03038

[65] N. J. Cherry, Human intelligence: The brain, an electromagnetic system synchronised by the Schumann Resonance signal, Med. Hypotheses bf 60 (2003), 843-844. https://doi .org/10.1016/S0306-9877(03)00027-6

[66] T. Nishimura, K. Mohri and M. Fukushima, The Mystery of the Dinosaurs: the Earth's Electromagnetic Field may Explain their Giantism and Extinction, Viva Origino 37 (2009), 7-9. 